\title{
Towards a Fluid Spectrum Market for Exclusive Usage Rights
}

\author{
Linda Doyle \& Tim Forde \\ Centre for Telecommunications Value-chain Research (CTVR), \\ Trinity College, University of Dublin, Ireland. \\ ledoyle@tcd.ie, timforde@mee.tcd.ie
}

\begin{abstract}
This paper focuses on highly fluid markets for trading exclusive spectrum usage-rights. The purpose of the paper is to underline the need for flexible usage-rights policies, as a core facilitator of such markets as well as to stress the need for a greater technical input to the debate. The paper builds on current work in the field of spectrum property rights and exclusive usage-rights. The first half of the paper captures the current state-of-play and presents a framework for visualizing the concepts involved. The paper goes on to make a clear distinction between the defining of a set of exclusive usage-rights and the exercising of those rights. This leads to a discussion of policies that are not alone about defining metrics and setting their desired values but are also about behaviors that involve negotiation and interaction. Through-out the paper the evolution of technology and its affect on the progress towards the goal of fluid spectrum markets is emphasized, as is the need for a very multifaceted approach to the challenges involved.
\end{abstract}

\section{INTRODUCTION}

In current spectrum management regimes, market forces have limited impact. There is however an emerging international consensus that market regimes will play much larger roles in the future of spectrum management and that a significant move away from the current $\mathrm{Com}$ mand \& Control approach is inevitable. The arguments for this move are well established and therefore not repeated in this paper and regulators such as the F.C.C. ${ }^{1}$, Ofcom ${ }^{2}$, and the E.U. ${ }^{3}$, have already indicated a move towards the use of more flexible trading regimes. Hence our starting point is that market-based approaches to

\footnotetext{
${ }^{1}$ Notice of proposed rule-making (FCC 00-402):promoting efficient use of spectrum through elimination of barriers to the development to secondary markets," Federal Communications Commission, November 2000 .

${ }^{2}$ Spectrum framework review statement, The U.K. Office for Communications, June 2005.

${ }^{3}$ The rspg opinion on secondary trading of rights to use radio spectrum (rspg04-54 final version)," E.U. Commission Radio Spectrum Policy Group, November 2004.
}

spectrum management will play a significant role in the future.

The potential market approaches that exist are varied. On the one side of the spectrum of options is the property rights/exclusive flexible use/licensed approach and on the other side is the open access/unlicensed/spectrum commons model. As Bill Lehr notes "Both of these cases are stylized as market-based because decisionmaking is decentralized to the market"[1]. There has been much debate as to the merit of these two extremes with economists such as Hazlett [2] favoring the an exclusive usage rights approach and Lehr [1] favoring a commons approach. In this paper we focus on exclusive usage rights. The paper is not about whether this is the sole and only reasonable approach, nor is it about the relative merits of this approach over the commons model. It is an attempt to tease out the issues involved in realizing a highly flexible and fluid exclusive usage rights market in which spectrum becomes a commodity that be can be traded and used as desired, within the boundaries of the rights associated with the commodity. The focus is on the very flexible types of policies that will be needed to underpin such a system.

The remainder of the paper is organized as follows. Section II captures the current state of understanding of the issues associated with the trading of exclusive rights and attempts to visualize the rights as applied to a very fluid market for trading spectrum. The basic time, frequency and space characteristics of the traded blocks of usage-rights are discussed. Section III builds on this and discusses the difficulties associated with exercising the actual usage rights that result from having to deal with electromagnetic radiation. Co-channel and adjacent channel interference are defined and visualized within our model framework and what we term the relativity of interference is discussed. Section IV then deals with the implications of section III through delineating the interference management options for two neighboring 
spectrum consumers, neighboring in terms of space or frequency. This section finishes by highlighting the need for interference management policies that permit a level of open negotiation between neighboring entities. In the conclusion the impact of technology on the evolution of exclusive usage-rights markets is discussed.

\section{The Starting Point; Visualizing And DEFINING THE USAGE RIGHTS}

Clearly, defining the rights associated with exclusive usage of spectrum is a challenging problem. There has been a range of interesting papers that have discussed this issue in the past number of years. Hatfield [3] does an excellent job of summarizing many of the main contributions to this debate and traces the development of the definition of spectrum usage rights through from Coase [4],[5], to De Vany [6],[7],[8], to Kwerel [9],[10],[11],[12],[13] and to Matheson [14], before going on to further the discussion himself. In the main, in all these papers, the approach has been to attempt to define the parameters associated with some kind of packet/bundle/block of spectrum with the end aim of making the definition tight enough

1) so that the user of the packet/bundle/block has a clear set of entitlements

2) and it becomes possible to trade a packet/bundle/block or multiples thereof in some kind of market system.

So for example De Vany talks about TAS packets (Time, Area, Spectrum). In this case the owner of the TAS-based rights would have the exclusive right to produce (information bearing) electromagnetic waves for a specified period of time (T), over a specified geographic area (A) and in a specified range of frequencies (S). Another example is the work of Matheson. He furthers the definition of the packet with his seven dimensions of electrospace that include frequency, the three dimensions of locationlatitude, longitude, and elevationtime, and the two possible directions of arrival (azimuth and elevation angles) and time. In one sense Matheson's definition is simply an expanding of the Area term by De Vany into a more rounded and complete definition that takes three dimensions into account.

In this paper we build on the work that currently exists and conceptualize what we call the radio spectrum rights continuum as a three-dimensional model: Space, Time and Frequency. Space, a broader term than Area captures the three dimensional notion of electromagnetic radiation We propose that such a continuum should be quantized into discrete packets/bundles/blocks. Each packet/bundle/block represents a unique assignment of spectrum rights at a particular place, for a particular frequency and at a fixed time. The purpose of quantizing the spectrum continuum on the time-axis is to allow each block assignment to be recycled and re-assigned at each time interval. For the purposes of consistency we will use the term block throughout the remainder of this paper rather than packet, bundle or any other such means of defining the discrete entity that has an associated set of usage rights.

\section{A. Usage Rights: A Visualization of the Present}

Though not used as a reference point for existing spectrum management regimes the RF spectrum rights continuum model can provide a helpful visualization tool, especially in terms of setting the context for future regimes. Hence Figure 1 attempts to visualize the access rights afforded to contemporary GSM and UMTS licensees in terms of Frequency/Space/Time blocks. A GSM/UMTS licence, under the model is constituted by a collection of unique Frequency/Space/Time blocks ${ }^{4}$. The allocations consist of spatially and temporally contiguous blocks of spectrum for a single frequency range which aggregate to form conventional licences, which reflects the underlying technologies and business models in play at the moment. In Figure 1 the red allocation represents the GSM license and the blue allocation the UMTS license, the latter license obtained by the licensee sometime after obtaining its initial GSM license. As shown in Figure 1, the allocations for the Republic of Ireland extend to the entire jurisdiction of the state - out to the southern, eastern and western coastal extremities of the island and to the border with Northern Ireland. The temporal element of spectrum rights rarely features in descriptions or charts of spectrum allocations and assignments, despite the fact that many licenses are for what de facto amounts to in perpetuity. The RF spectrum rights continuum model therefore emphasizes this long-lived-ness and the allocations extend to the future indefinitely.

Figure 1 also attempts to capture the static nature of the current system. The UMTS license in Figure 1 is obtained at a time in advance of a $3 \mathrm{G}$ market emerging as has been the case for most operators. It should also be noted that Figure 1 embraces no notion of liberalization

\footnotetext{
${ }^{4}$ Note however in the diagrams used in this paper, for simplicity, space - whether two-dimensional ( $\mathrm{x} \& \mathrm{y}$ ) or three-dimensional ( $\mathrm{x}, \mathrm{y}$ $\& \mathrm{~h}$ - height above ground) - is collapsed to one-dimension in this model.
} 

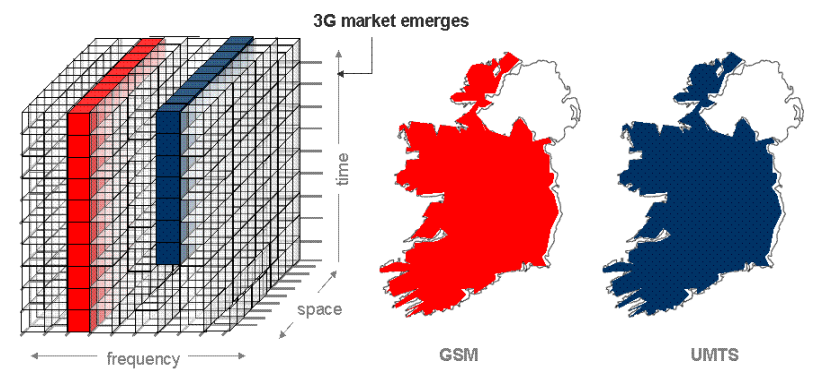

Fig. 1. Representations of an Assignment for a Single GSM/UMTS Network Operator

It important at this stage to also point out that the use of Ireland as a reference point and the size of the individual cubes in the diagrams are for illustrative purposes only and are not meant to actually suggest size in terms of frequency, space or time. The actual size of the blocks is an open question.

\section{B. Usage Rights: An Ideal Visualization of the Future}

Moving on from the visualization of current regimes we can now use the RF spectrum rights continuum model to visualize the future. At its most extreme, our proposal for a flexible market would facilitate a radical form of disaggregation of the RF spectrum, which could have a similar effect on the wireless telecommunications industry. This is illustrated in Figure 2. The Rubik's cube-like illustrations represent an area of the spectrum rights continuum in which there has been complete disaggregation of assignments over frequency, space and time.
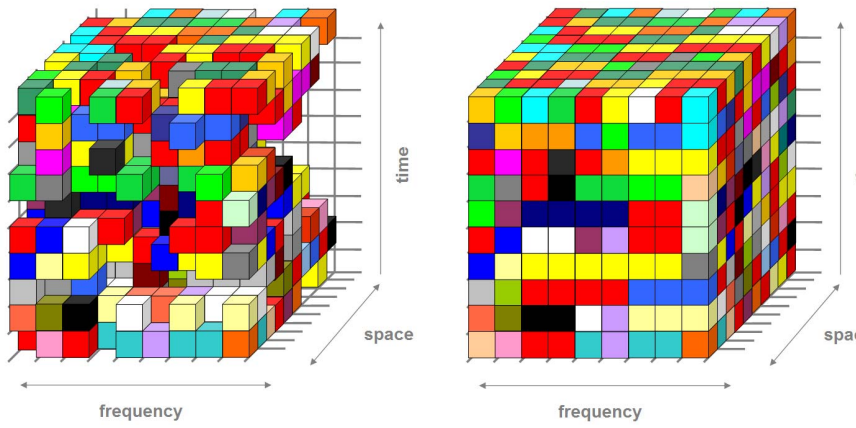

Fig. 2. Completely Disaggregated Market-based Assignments

\footnotetext{
${ }^{5}$ At the outset, it should be clarified that the term spectrum assignment is taken to mean assigning spectrum to a particular user (e.g. network operator) and spectrum allocation is understood to mean allocating spectrum for a particular use or service, i.e. non liberal usage. Hence we use the term allocation in the context of existing regimes and assignment in the context of future regimes.
}

Each block in the Rubik's Cube like figure represents a unique spectrum assignment and each color represents a unique spectrum consumer. We have chosen the term spectrum consumer to convey the point that we do not wish to pre-empt the nature of future spectrum users or businesses built around fluid spectrum. Rather, we consider that some agent will want to acquire spectrum for some, possibly still unforeseen, use. We currently would understand such a market actor in terms of entities such as cellular network operators, TV companies and wireless broadband providers.

In a very fluid and flexible market spectrum consumers can freely buy and sell the exclusive rights to the cubes of spectrum that are illustrated in Figure 2. The services that owners of the usage rights deliver and the technologies used to deliver those are not proscribed, i.e. there is total liberalization of the spectrum. There are no limits or rules as to what blocks can be neighbors and what services can be delivered by neighboring blocks.

In such a very fluid system, the individual blocks of course may be aggregated to form larger assignments. This is depicted in Figure 3. Aggregations in this situation occur because of market drivers and not because of limitations due to the system for acquiring access to spectrum. Figures 2 and 3 therefore attempt to capture the fluidity of reaction to market forces that would characterize our framework.
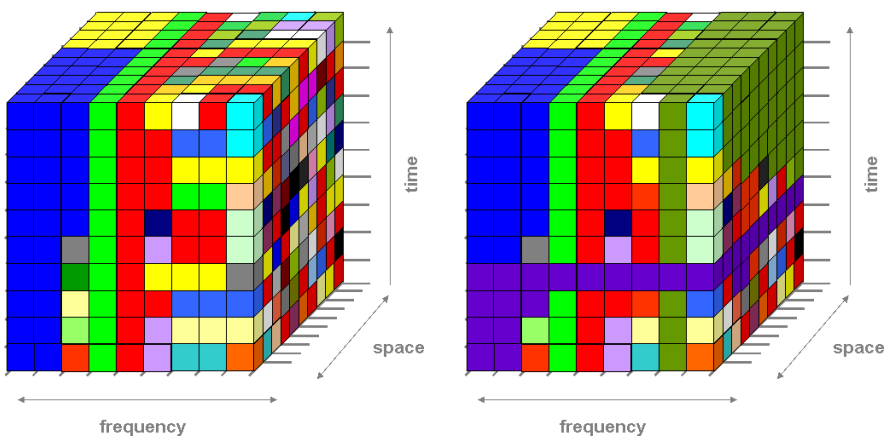

Fig. 3. Completely Disaggregated Market-based Assignments

\section{The Dimensions of the Frequency/Space/Time Block}

The use of uniform cubes in the Figures 1, 2 and 3 is a conceptual starting point ${ }^{6}$. As emphasized in the previous section, these figures present an idealized view

${ }^{6}$ In terms of space the rectangular (or square) shape is accepted as feasible. While the footprint of radio signals is often depicted by hexagonal shapes in the literature, the Australian authorities determined that such a representation was neither especially accurate nor practical from the point-of-view of assembling larger space-rights packages. 
of the notion of spectrum as a commodity. The realities of dealing with spectrum as a tradable commodity will in fact be dealt with in sections III and IV. However for the purposes of the current discussion, it suffices to continue focusing on the frequency/space/time block in order to pose a number of questions relating to its general dimensions.

The frequency/space/time dimensions of the blocks may take a different shape and may be non uniform in size. It may be desirable to have one base block size from which commonly traded units emerge. In this case the idea would be to use the smallest frequency/space/time that makes sense and to build all trading packages from this. Or it may be preferable to have heterogeneous block sizes, made from a range of discrete acceptable frequency, space and time dimensions ${ }^{7}$. For the purposes of this paper we will focus on the spatial dimension and explore the factors that might influence it's choice but a similar analysis could be performed for the other dimensions.

1) Block Size - Spatial Analysis: In terms of creating a basic block size, consider the following spatial analysis, depicted in Figure 4.

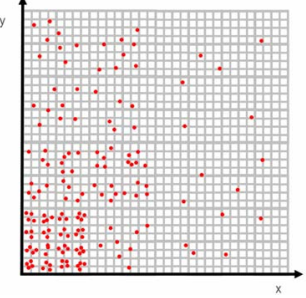

(a) Population Density Spread for Area Under Consideration

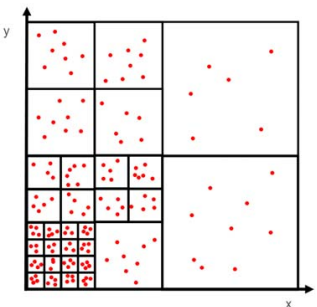

(b) Space-Right Unit Agulation Densities gregations Based on Pop-

Fig. 4. Factors Affecting the Aggregation of Space-Right Units

We consider the requirements of a spectrum consumer, e.g. a network operator, that decides that it would be economically beneficial to build-out a network in increments that serve no less than five to eight population clusters. In this case the decision of the network operator could be driven by the cost of existing radio and network technologies, by the nature of the intended service (e.g. digital TV, Broadband Internet, etc.) and by the cost of spectrum suited to these technologies and services. Figure 4(a) illustrates the spread of population density over that area; each red dot represents a population cluster of,

\footnotetext{
${ }^{7}$ In terms of frequency the Australian model sets basic frequency rights dimensions as low as $0.0125 \mathrm{MHz}$ for some frequency ranges with dimensions increasing to as big as $\mathrm{XMHz}$.
}

say, one thousand people. The spectrum consumer can then decide that it is only worthwhile for it to package the basic space-right units according to this population information so that it does not arrive at larger spaceright units than it deems economically viable. So in Figure $4(b)$ the smallest block dimension is evident on the left hand corner and the largest traded entity, on the top right hand corner, is a multiple of the basic block size.

The main point to take from this example is that it is necessary to ensure that the basic space-rights units are initially defined as small as possible so that a potential spectrum consumer can assemble a package of rights that just meets its requirements. Spectrum will be wasted if a consumer has to acquire excess spectrum rights just because the basic space-rights size was initially set too large. The second point to be taken from this example is that while the example is based on current costs of a given network operator and the current technological characteristics of those networks, basic block sizes should not be chosen to only reflect current technological capabilities. So while contemporary networks range in coverage from country-wide (cellular), to city-wide (WiMAX) to LANs (WiFi) and PANs (Bluetooth), a somewhat future-proofed system should not pre-empt technological and economic advances by basing its definitions of spectrum rights units solely on existing standards.

2) Combinations of Blocks - Spatial Analysis: The basis block size should also perhaps be considered in the context of the framework for trading as well. Following on from the population based example for determining basic units, we can consider the trade-off between the flexility afforded by the definition of very small units and the complexity introduced by creating too many units as it may prove burdensome to search through and package such basic rights. A lot therefore depends on the combinatorial options within the trading framework and how easy they are to determine and to subsequently bid for and acquire. To put this in context consider, the following second example that focuses on space dimensions, which walks through a potential process involved in how a spectrum consumer might go about assessing its spectrum rights requirements and assembling a desired package.

Figure 5(a) illustrates a sample terrain over which a network operator wants to establish a network based on a single-transmitter network, say for a single DVB-T cell. In this case, the spectrum consumer has many variables to consider such as those associated with antenna choices 
(power, polarization patterns, height above ground, etc.) and with the terrain (elevations, typical climate etc.). Associated with this terrain would be the grid of basic space-rights as depicted in Figure 5(a).

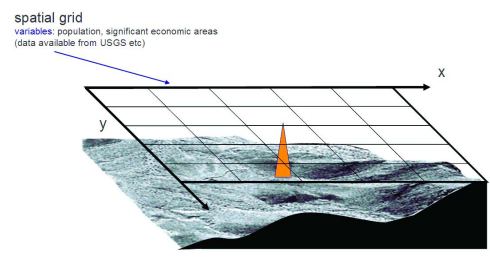

(a) Terrain Model with Basic SpaceRights Grid Overlaid

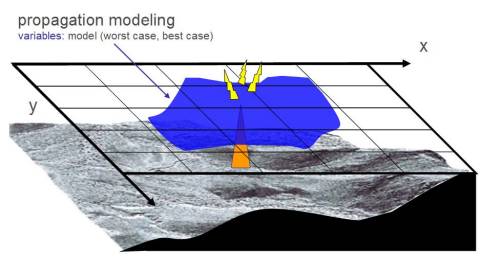

(b) Basic Space-Rights Grid with Worst-case Propagation Model Overlaid

Fig. 5. Fitting Worst-case Spectrum Requirements to Space-Rights Grid

Using propagation modeling software, the spectrum consumer can arrive at a best-case/worst-case coverage footprint for its intended network. Such a footprint, as depicted in Figure 5(b), would outline the extent to which the radio signal propagates at a level which exceeds the maximum noise threshold. Once the potential network operator has assessed its propagation footprint, it maps it to the the frequency/space-rights model, as shown in Figure 6(a). Finally, the spectrum consumer is able to determine the combination of blocks of rights that it must acquire to operate its network, as depicted in Figure 6(b), and knows what it needs to acquire in the market.

It could therefore be argued, that whatever market framework exists for trading in blocks, the ease in which combinatorial bids can be made is a factor which also may determine the smallest unit that can viably exist. The transaction costs for small units and the transaction costs associated with larger packages of blocks need to be assessed as the value of the trade must be substantially greater than the transaction costs. To further complicated the problem, the actual technology that is used to perform the trades may also play into this equation. So for example a fully automated cognitive trading system that automatically maximizes options for the traders may lend itself to trading in block sizes comprising the smallest block unit, whatever that may be, while a more

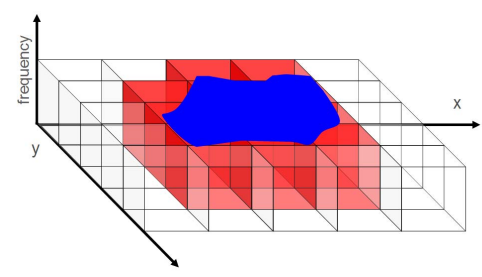

(a) Mapping of Worst-case Propagation Model to Spectrum Units

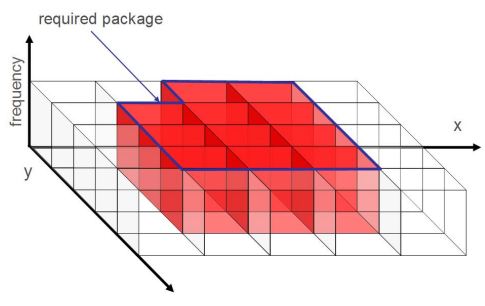

(b) The Required Package of Spectrum Units

Fig. 6. Worst-case Spectrum Package Requirements for Single Network, Single Transmitter Case

traditional paper-based and slower system may lend itself to trading in hundreds of units at any one time.

Overall however it is important to create a flexible enough system so that whatever commonly traded combinations are useful, can emerge. One can envisage a situation where at different frequency ranges, while the possibility of trading in small units theoretically exists, that combinations of less than X Blocks are never traded and that de facto trading sizes come into being. One can also envisage a system in which what is a de facto trade size changes as technology changes over time.

3) Technology Advancement - Spatial Analysis: Continuing along the lines of the technological influences on the block size, there are some communication system technological limits to realizing each of the frequency/space/time dimensions. Any consumer of a block may deem to divide and sublet that block and undoubtedly technological barriers will be encountered at some stage of that division process. Figure 7 attempts to capture the subletting concept. As the sublet blocks get smaller, technical bottlenecks may arise.

However, this paper focuses on the primary (meaning main in this case) trading market for spectrum, and is not concerned with the subletting process and hence it is not envisaged that technical limits will play a major role in this overarching fluid market structure. Although, it should be noted, that by not proscribing in advance the degree to which subletting can occur, the possibility of allowing future communication system advancements to be naturally incorporated into the system as they arise, 


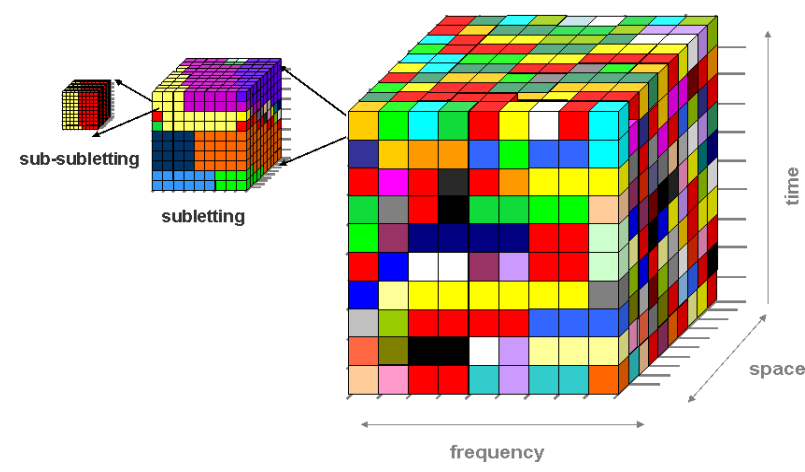

Fig. 7. The Subletting Process

is strong.

Figure 7 is also useful in terms of conceptualizing who the traders will be. In the examples in the previous sections, a network operator like entity was considered to be the first level of trader. While we advocate this level of access, i.e. access by any network entity to the purchase of spectrum usage rights, it is still an open question as to who would trade.

\section{But this is just an Idealistic View?}

The discussion thus far has been general and idealistic in order to present an the overarching view of a fluid spectrum market and to focus simply on the frequency/space/time spectrum block of spectrum usage rights. Even at this simple stage broad questions about the dimensions of these blocks can be posed and it is possible to begin envisaging frameworks within which these blocks could be traded. The more difficult analysis begins now. The somewhat ideal concept images of Figures 2 and 3 come up against serious problems when it comes to the exercising of the rights associated with each individual block. In fact it can be argued the defining of usage rights is not the problem but in fact the defining of how to avail of those rights is.

\section{The Problem of EXercising Your Rights TO THE FREQUENCY/SPACE/TIME BLOCK}

A large number of papers have been written that address the issues involved and provide useful background material. In a nutshell the problem is that electromagnetic waves cannot be fenced in and hence spillage beyond the boundaries of the blocks occurs. Hence exercising your rights to your block in such a manner so as not to infringe on the rights of others can be extremely tricky. Not alone can the waves not be confined, it is difficult to determine by how much a given signal goes beyond a boundary as the actual distance which a given signal can travel can vary. Depending on frequency, the waves propagate in different ways, making use of the ground or the atmosphere, can be reflected from multiple surfaces such as buildings or landscape features and/or refracted and bent around obstacles. Signals can propagate different distances depending on time of day, weather conditions or the season. The signals from any one block therefore have the potential to interfere with signals in other blocks. The are no simple fences that can be erected to keep the signal within the frequency and space boundaries associated with the block of usage rights. This means that typically at the edges of a spectrum block, the signal will not be zero, i.e current technology cannot produce a signal that forms a nice neat cube shape. The impact of this is when it comes to exercising the rights, is that frequency, space and time are not adequate for defining the block of rights fully and some notion of signal behavior at the boundaries must be introduced.

\section{A. Co-channel \& Adjacent Channel Interference}

In technical terms the spillage beyond the boundaries of the block manifests itself in two ways, as co-channel interference and as adjacent channel interference.

Co-channel interference may be experienced by networks that are operating on the same frequency but whose transmitters are not sufficiently physically separated or whose transmission power is too high. Figure 8 depicts two slices of spectrum consumers in the RF spectrum rights continuum, that are at any time interval using the same frequencies in different locations. When one spectrum consumer owns the entire slice, as in the case of the red colored block in Figure 8, the positioning of transmitters can be collectively chosen so not to cause co-channel interference and at the same time to optimize the capacity of the network. This leads to, for example, a cellular network planning in which neighboring basestations operate on different frequencies so as not to interfere, while more remote basestations can then re-use the same frequency (there is what is known as a frequency reuse distance). Hence users A and B from the red slice in Figure 8 will have collaboratively divided up the range of frequencies available within the blocks, so that they are not using the exact same frequencies from the range that they are entitled to use.

In the fluid spectrum market that we advocate, such collective planning is not always an option. In other words users A and B from the multiply owned slice in Figure 8 will not tend not to be part of a co-operative network planning scheme. The way 


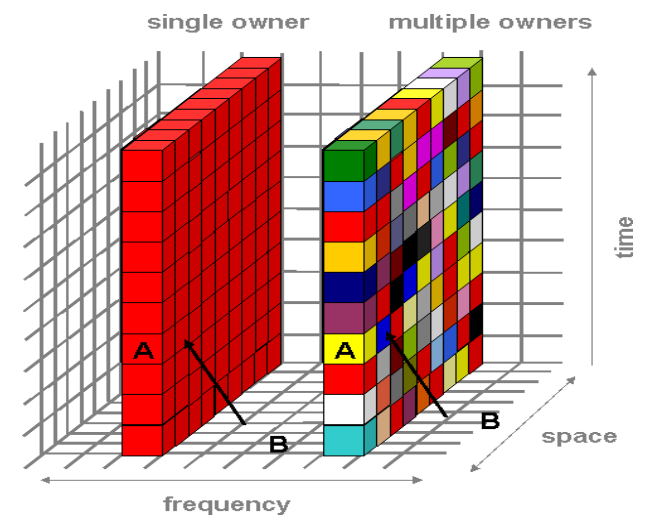

Fig. 8. Co-channel Interference

to ensure that co-channel interference does not happen in this case, is by specifying the power levels that should be met at the boundaries of the block. White's [15] phrasing comes in useful here. He speaks about spectrum-band/area/beyond-perimetersignal-strength-limit/time-period "parcels". Changing "area" to "space" to take the fuller three dimensional nature of the signals into account and using our phraseology, we now can speak about frequency/space/beyondspace-signal-strength-limit/time-period block.

Adjacent channel interference occurs when power from one frequency channel leaks into an adjacent frequency channel. Figure 9 depicts two slices of spectrum consumers in the RF spectrum rights continuum that use adjacent frequencies within the same space. As with co-channel interference, adjacent channel may cause too much noise at the receiver, such that the receiver cannot decipher incoming signals. The slice of spectrum consumers towards the front of the diagram, attempts to capture the current regime which consists of a limited and controlled number of spectrum consumers, each of which is only free to use very specific technology to deliver very specific services. Because of these restrictions, it is possible to regulate for interference through the use of predefined guardbands in which no spectrum consumers are allowed. The width of the guardbands are known, as the regulators will have decided in advance what technologies and services can be neighbors and hence the amount of space (in frequency terms) needed between each.

In the spectrum market we advocate, there are no rules governing what services should be delivered by neighboring blocks and what technologies should be used to deliver them. So users A nd B in the background slice in Figure9 can deliver any services

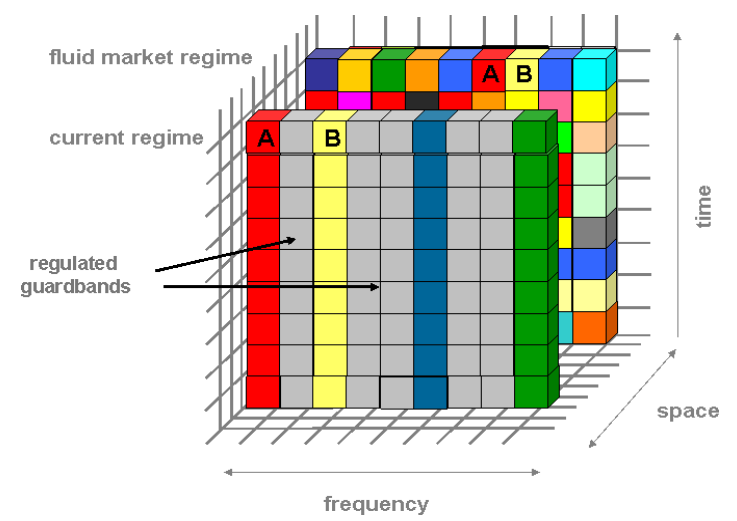

Fig. 9. Adjacent Channel Interference

they so choose, using any technology they see fit and hence predefining guardbands is not an option. Therefore, just like in the co-channel case we need to specify boundary conditions, this time in terms of frequency. Using the phraseology from above we now speak about a frequency/beyond-frequency band-signal-strength-limit/space/beyond-space-signalstrength-limit/time-period block. Users must respect these boundaries in order to avoid adjacent channel interference.

\section{B. The Theory of Interference Relativity}

The previous discussion emphasizes the need to define a frequency/beyond-frequency band-signal-strengthlimit/space/beyond-space-signal-strength-limit/timeperiod block. However what it does not fully capture is the relativity of the situation at the boundaries. To understand this point consider the two cases of neighbors illustrated in Figure 10. The top two neighboring houses of Figure 10 are owned by two individuals comprise up Scenario 1. The individual in the left hand house is deaf and the individual on the right hand side plays excessively loud music. The playing of the music does not interfere with the deaf owner of the neighboring house.

The bottom two houses in Figure 10 are owned by two other individuals and comprise Scenario 2. The individual on the left has built an array of highly sensitive microphones around his property. The individual on the right plays music at a low level but because of how the neighbor has constructed the house, the individual is deemed by that neighbor to be causing unacceptable levels of noise.

In both these cases, the perceived interference is a combination of the circumstances at the boundaries. It may be argued that the circumstances at the boundary 


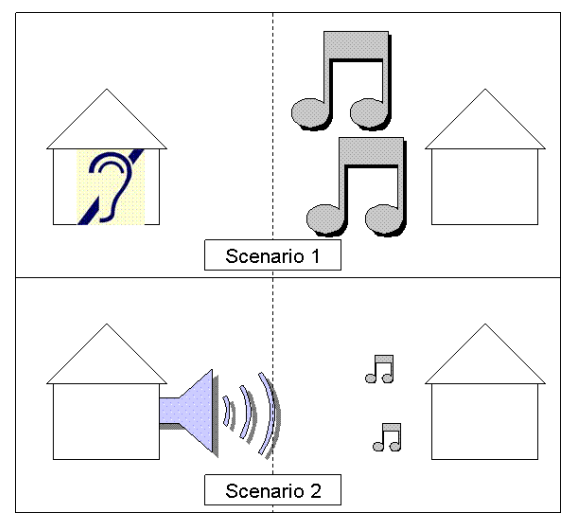

Fig. 10. Relative Interference

in Scenario 1 is lucky for the neighbor playing the loud music. It may also be argued that in Scenario 2 it is unreasonable that the individual in the house surrounded by microphones can deem the neighbor to be interfering. The scenarios here appear as two extremes but in fact not that far removed from reality, as will become evident in the co-channel and adjacent channel examples below. The key point for now is that the exercising of the rights associated with the frequency/beyond-frequency band-signal-strength-limit/space/beyond-space-signalstrength-limit/time-period block, even in the scenario where metrics for limits at the boundaries have been establish can be complicated. In other words the story does not end with the defining of the frequency/beyondfrequency band-signal-strength-limit/space/beyondspace-signal-strength-limit/time-period block.

\section{X Marks the Spot}

The spreading of electromagnetic waves beyond a defined boundary occurs in a complex manner as can be seen from the previous discussion. Figure 11 attempts to bring the various concepts together. In this Figure the Red Block depicts the rights of a given spectrum consumer at a certain time, for a given frequency range and spatial extent. The area shaded in dark grey represents the potential for interference (i.e. whether it be via co-channel or adjacent channel interference). Please note again that the cubic representation is not meant to suggest the radiation patterns are in neat cube formats but are used merely to delineate a volume of interference irrespective of what the exact shape will be.

The extent of the grey area is then dependent on the actual physical propagation characteristics of the electromagnetic radiation at the frequencies in question which in turn can be dependent on the characteristics and settings of the transmitters, the terrain, object move-

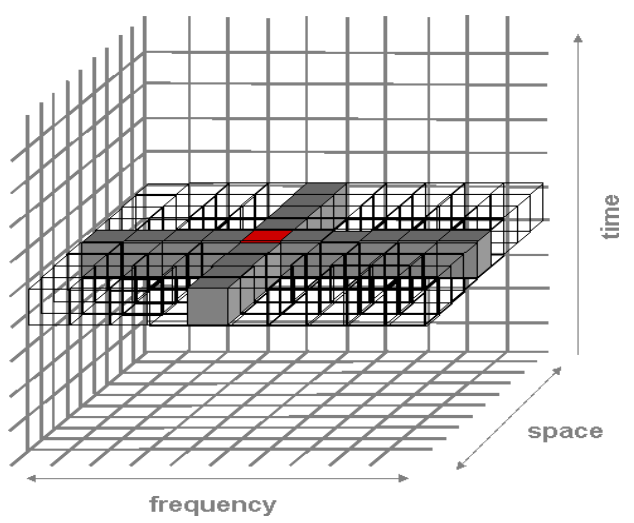

Fig. 11. The Affect of Interference: The Usage Rights are marked in Red. The area shaded in Dark Grey depicts the Potential for Interference

ment etc. in the area associated with the usage rights combined with the characteristics and settings of the receivers in the area outside of that associated with the usage rights.

Viewed in this way we can conclude that the exercising of the rights associated with a given block involves the interactive creation or forming or constructing of a frequency/beyond-frequency band-signal-strength-limit/space/beyond-space-signalstrength-limit/time-period block. In other words we need interference management policies that are not alone about metrics and their desired values but are also about block construction behavior.

\section{Active Interference Management APPROACHES}

The notion of the interactive creation or formation of the frequency/beyond-frequency band-signalstrength-limit/space/beyond-space-signal-strengthlimit/time-period block is best illustrated by returning to a discussion of co-channel and adjacent channel interference in order to tease out the options for spectrum consumers.

\section{A. Co-channel Interference and Options}

Consider now two users A and B operating at the same frequency but separated in space, from the fluid market diagram in Figure 8. Assume now that user B is not remaining within its frequency/beyond-frequency band-signal-strength-limit/space/beyond-space-signalstrength-limit/time-period block and its signal strength beyond its space is exceeding the required level and interfering with user A, i.e. user $\mathrm{A}$ is experiencing co-channel interference. The following are potential solutions to this problem: 
1) User B reduces its power significantly so as to decrease the boundary power levels and be sure it is meeting its boundary conditions for the worse case scenario. Some of the factors that might be adjusted to minimize interference include the antenna height, antenna gain, antenna orientation, transmitter and antenna location and transmitter power. It may be necessary to try a combination of several of these methods to effect a satisfactory solution.

2) User B decides to use technology that combines real-time sensing techniques with cognitive functionality to monitor and adjust in real time the power levels at the boundaries rather than just dealing with the worse case scenario.

3) User B gets user A to agree it does not matter.

4) User B makes an offer to User A and buys out the usage rights to the spectrum block currently owned by User A.

5) User B gets user A to buy it out.

6) User A / User B find an alternative solution we have not yet envisaged

The solutions listed above, may or may not be economically viable. The technical solutions have costs. The blunt lowering of the power may have the overall affect of reducing coverage and losing customers. The sophisticated and dynamic power adjusting scheme, while not blunt, may be currently hugely costly to install. The bargaining with other users that leads to the buying out of one or other of the users may be prohibitive both in terms of transaction costs as well as in terms of actual purchasing of the rights. There may be game playing involved, for example on the part of user B, to force a buy-out. The option for getting user A to say it does not matter would is akin to Scenario 1 in Figure 10 where user B is the loud music playing neighbor and user A is the deaf neighbor. Of course the ownership of the rights to block A can change and the solution that B gets A to say it doesn't really matter, becomes null and void.

Hatfield's and Jackson's comments on the lack of suitability of the AM frequencies as tradable commodities can be revisited in terms of the listed suggested solutions [3],[16]. Hatfield states "For a band like that traditionally used for AM broadcasting, it seems totally impractical, if not impossible, to provide licensees with anything close to certainty in terms of interference protectionat least using the classic property law trespass concept. If, for example, a station in an adjacentor more remotegeographic area could prosecute a trespass claim against a transmitter that created interference, it could seek dam- ages or injunctive relief based upon a series of natural conditions that happen only infrequently. Jackson who is also of this mind believes that the cellular and PCS bands are more amenable to a property rights regime because of limited signal range, systems operating over large blocks of spectrum and over large geographic regions, and control over both transmitters and receivers by the system operator.

Using the list of solutions above and applying them to the AM case, it would, for example, be possible to use adaptive systems to manage the day/night propagation characteristics of the AM waves. Alternatively solution 3 , in which A gets B to say it does not matter can be nicely illustrated with an AM scenario. Supposing user B is a 24 hour Radio Station, while A is a daytime only Radio Station. The longer propagation of B's signals during the night will have no effect on A. Failing either of these options, the buying of more usage rights blocks might make most sense. In fact what may emerge is that certain frequencies will end up being sold in combinations of certain numbers of blocks and never in individual blocks units, along the lines of the discussion in Section II-C

It is clear that a very fluid market for spectrum usage rights trading is indeed quite a complex entity. However it is clear that solutions to the co-channel problem exist. Whether all options are currently economically viable or currently completely technically feasible should not be seen as a barrier to the advent of such markets but the broadening of the solution space over time should be built into the system.

\section{B. Adjacent Channel Interference and Options}

Consider now two adjacent frequency users, A and B, in the fluid market part of Figure 9. Supposing now that power from the frequencies used by user B leak into the frequencies used by user $\mathrm{A}$ and cause interference above an acceptable level. The following is a list of potential solutions to this problem:

1) User B improves the spectral masks it is using in its system (From a network operator's point-ofview, out-of-band-emissions can be minimized by the use of appropriate transmission masks. Such masks have the purpose of shaping the signal as it leaves the transmitter, blocking or reducing power leakage to adjacent frequencies. A transmission mask will guarantee that within some $\mathrm{X} \mathrm{MHz}$ of the desired signal bandwidth that the leaked power will taper off to an acceptably low limit). 
2) User B creates a self-imposed guardband between it and user $\mathrm{A}$

3) User A creates a self-imposed guardband between it and user B

4) User B and User A both create self-imposed guard bands.

5) User B gets user A to agree it does not matter.

6) User B makes an offer to User A and buys out the usage rights to the spectrum block currently owned by User A. This effective allows use A to encroach on frequencies originally owned by user B.

7) User B gets user A to buy it out.

8) User A / User B find an alternative solution we have not yet envisaged

The solutions listed above need perhaps a little explanation. For example the self-imposed guardband should be explained. Under our proposal, we would like to see a situation in which the spectrum consumer exercises more choice over whether it wants to acquire excessive bandwidth rights and operate cheap radios with leaky transmission masks, or spend money on more expensive transmission masks so that it can save on spectrum costs. Figure 12(a) depicts the two consumers setting up a service within each of its exclusive usage-rights block with each consumer choosing to impose different guardbands so as not to violate its neighbor's rights.

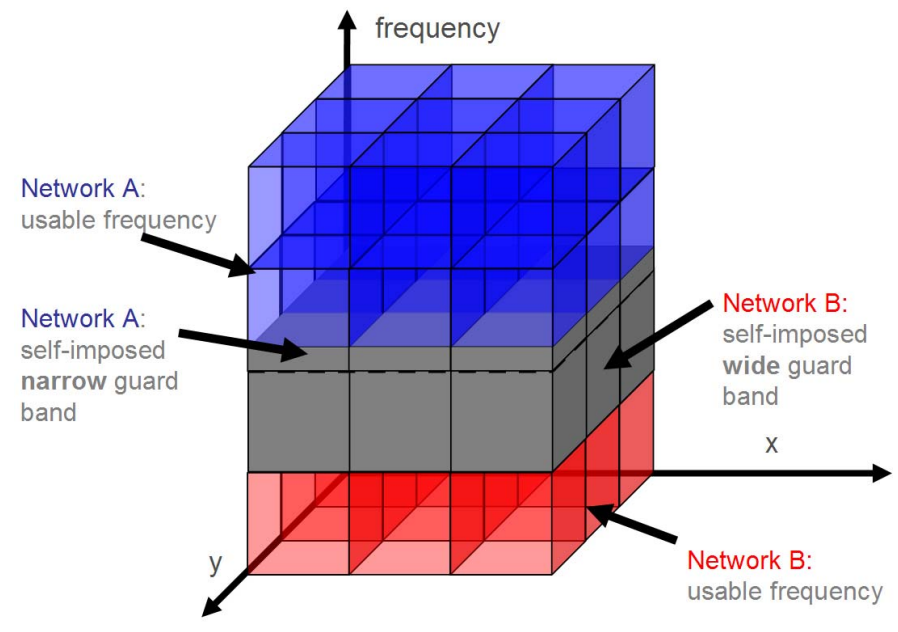

(a) Self-imposed Wide and Narrow Guard Band

Fig. 12. Variable Guard Bands

The options for the creation of guardbands in the solution listed above cover all potential reasons interference scenarios. So for example if B is actually the cause of the interference it alone can use a self-imposed guardband. If however A has chosen to use over- sensitive receivers and is picking up stray frequencies more readily, much like in Scenario 2 in Figure 10, then A will have to readjust its operating frequencies and use a self-imposed guardband too. This would be akin to the owner of the house surrounded by the sensitive microphones moving the microphones indoors for example. Alternatively as in Figure 12(a) both parties can choose to use them.

The deployment of very good masks is a valid option option. An interesting finding of a recent study of the problems posed by the adjacent placement of UMTS and DVB-T systems was the fact that standard, off-the-shelf DVB-T transmitters were now meeting the critical cases mode mask of the DVB standard [17], as shown in Figure 13. The critical cases mode mask is mandated for DVB transmitters that are adjacent to very senistive systems, above and beyond the level of care needed to site a DVB service next to an UMTS service. This study shows that even at this early stage, radio equipment is meeting and exceeding some of the challenges necessary for the fluid assignment of services in adjacent bands.

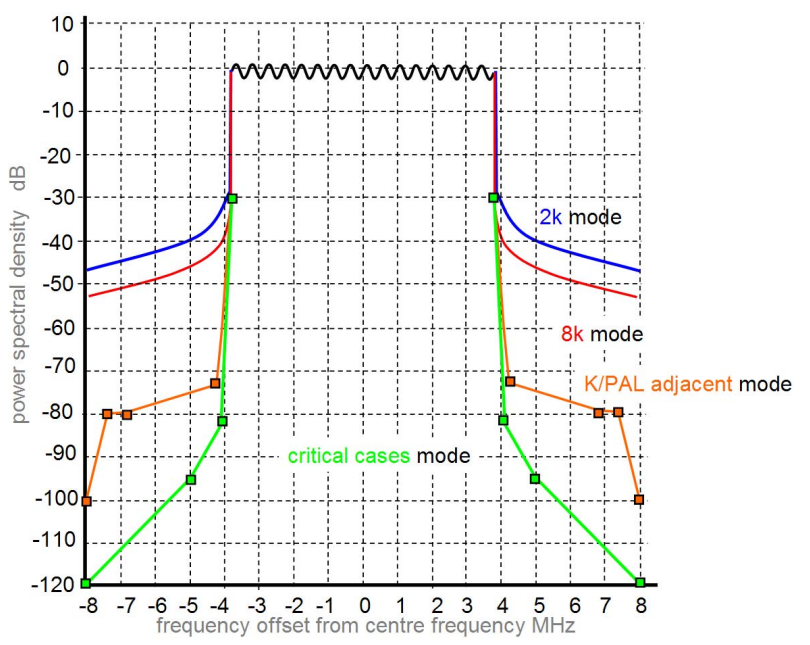

Fig. 13. DVB Masks

As in the case for the co-channel interference, the solutions listed for the adjacent channel interference, may or may not be economically viable. The technical solutions have costs. So for example, as with most technologies, the better the mask, the more expensive it is. The bargaining with other users that leads to the buying out of one or other of the users may be prohibitive both in terms of transaction costs as well as in terms of actual purchasing of the rights. Again there may be game playing involved, for example on the part of user B, to force a buy-out. Of course again the rights to block A can change ownership and the solution that B gets A to say it doesn't really matter, becomes null and void. 
However, it is worth stressing that while we recognized that the situation is hugely complex, we also recognized that the problems are solvable and that as time advances the solution space may even grow.

\section{Discussion}

While the previous case studies were illustrative there are two distinctive points that must be made.

Point 1: The Essential Move from Inputs to Outputs: Firstly in discussing the possible solutions for the co-channel and adjacent channel interference problems in the previous sections, we embraced the ideas of De Vany when he calls for a shift from prescriptively regulating practices and activities (e.g., individual transmitter locations, powers, and antennas antenna heights) to focusing only on the desired result (e.g., the strength of the signal at the boundary). The reality of this is that the consumer of the usage rights block gets to decide what exactly suits it best. In other words the onus is on the exerciser of the rights to behave in whatever way is most suitable to achieve the goals of staying within the boundaries of its rights, be that in terms of frequency, space, time and signal strength. In the examples in this section the spectrum consumer of the block makes choices as the consumer sees fit. The idea of the construction of the block of rights and the idea of the need for policies that should be associated with construction behaviors, encapsulates this ethos.

Point 2: The Need for Open Negotiation: The second point worth stressing is that the above list of solutions also encompasses some notion of interactive behavior across boundaries, what we are calling open negotiation. On one level this is not such a huge step from what currently happens, albeit in a slow and cumbersome manner, for example when for example operators inadvertently encroach on each others territories and come collectively to some kind of solution. It also has echoes of what happens when bilateral roaming agreements are hammered out between different operators. On another level it is a huge move forward to a more sophisticated engagement with the nuances of a fluid market. Open negotiation can be thought about on multiple levels, ranging from more defined technical approaches to more complex economic scales. On the broader front, Forde [18], in a companion paper to this, explores the concept of Coasean Bargaining and offers a framework within which many of the types of negotiations mentioned here occurs. This need for open negotiation further underlines the need for polices that encapsulate behaviors and are flexible.
The key point to take from this discussion is that policies that are flexible are needed to facilitate fluid and dynamic spectrum markets. The need to be flexible so that a spectrum consumer can exercise choice on how it meets it obligations as a spectrum user. They need to be flexible to allow an element of negotiation feature in the system. Whether negotiation occurs before or after the trade is an open question. From an engineering point of view trading with no regards to who your neighbor is or might be in the future, is the more attractive solution (with the possibility of automated cognitive negotiation becoming a feature of networks of the future). However negotiations in advance of trading may prove economically viable.

\section{CONCLUSION}

This paper is the beginning of an argument for the design of policies that can encode both the interactive process involved in exercising of rights and the desired outputs of that process. By this we mean that exclusive usage rights are all about the exercising of those rights through the sculpting and shaping of the the electromagnetic frequency/beyond-frequency band-signal-strength-limit/space/beyond-space-signalstrength-limit/time-period block so as not to infringe on others (or for them to infringe on you). A large body of work is needed to further progress the ideas in this paper. In particular, it is necessary to bring the actual impact of evolving and emerging technologies on an exclusive usage-rights market into the discussion. In the concluding sections of the paper we therefore lay the groundwork for further research. There are three technological influences that must be considered ${ }^{8}$

\section{A. The Technologies of Electromagnetic Sculpting}

The first set of technologies are those associated with exercising of exclusive usage rights. This essentially means technologies that facilitate the sculpting and shaping of the the electromagnetic frequency/beyondfrequency band-signal-strength-limit/space/beyondspace-signal-strength-limit/time-period block so as not to infringe on others. Hardware that provides good filtering to stop frequencies leaking into other bands is a straightforward example of a technology that helps shape the usage-rights block. Complex technologies that would allow neighboring (in frequency or space) communication systems to automatically negotiate with

\footnotetext{
${ }^{8}$ Note that the term technology is used in a very broad sense and generally refers to hardware and software techniques, methods, approaches, algorithms or solutions.
} 
each other along the lines described in the paper thus far or approaches that allow radios to read from a database of usage rights consumers and accordingly adjust their radiation patterns to take neighbors into account, are all included here. The development of metrics such as the interference temperature, the use of interference temperature services (e.g. a sensor network that provides spatially distributed interference temperature data), the design of clever sensing techniques that contribute to being able to sense the strength and pattern of the radiated signals and hence inform the process of electromagnetic sculpting are other examples. Note that Interference Temperature metric was introduced by the FCC. Kolodzy [19] provides details of this approach to interference management. Adaptive beam forming, adaptive power selection etc. all form part of the approach to shaping a signal to make best use of the usage rights so as not to infringe on others. Included also are all techniques and approaches which not alone form the radiation pattern associated with the shape of the block but also are focused on extracting maximum capacity from the block in terms of users who have access to the spectrum.

\section{B. The Technologies of Spectrum Fungibility}

The second set of technologies are those associated with the fungibility of spectrum. Fungibility is a measure of how easily one good may be exchanged or substituted for another example of the same good at equal value. In the case of spectrum we are interested in how easy it is to exchange or substitute the the spectrum used in delivering a service from one range of frequencies to the other. This is a very important concern. The use of software and reconfigurable radios will for example play a role here. In an ideal world, these kinds of radios and devices would have wideband frequency agile frontends covering the whole electromagnetic spectrum and and be capable of reconfiguring to use any available spectrum. At the terminal end it is possible to image users being able to reconfigure to avail of whatever service needed using whatever spectrum is available. When thinking of the general infrastructure associated with many networks however being fungible is challenging. So for example a cellular operator offering a service that covered a particular area $X$, with a particular number of basetations $Y$ and a given range of frequencies $Z$, would have to cover the same area with the same service were the frequency range changed to $Z 2$, for example, even though this might necessitate a change in numbers of basestations from $Y$ to $Y 2$, where $Y 2>>Y$. It may be that this way of thinking about network infrastructure and service delivery will become obsolete but it is likely that fungibility will only increase to a point and possibly only apply within groups of frequency ranges.

\section{The Technologies of Trading}

The final set of technologies that will play a role in the evolution of exclusive usage-right markets are the technologies associated with the trading itself. So for example, whether the trades are carried out using a paper based mechanism or whether a fully automated market in which software agents compete for spectrum for the spectrum consumers is used, will have an influence as these issues may be interlinked with the transaction costs that might occur. There are many kinds of transaction costs. For example search and information costs arise in determining that the required good is available on the market, who has the lowest price, etc. Or bargaining costs are the costs that are incurred when coming to an acceptable agreement with the other party to the transaction, drawing up an appropriate contract and so on. The technology involved in these costs will play a role as the ease and the speed at which these transactions can occur will influence the dynamics of the market as well as the size of the trading units.

\section{In Summary}

There is an intensive margin in exercising the usage rights measured by the cost of the technologies needed to do to squeeze the maximum usage from those rights while managing interference. There is an extensive margin measured by the cost of making frequencies more fungible. The interplay of these margins needs to be further investigated as does the impact of the technologies of trading. In a somewhat contradictory manner, technology neutral policies that will not hamper innovation and progress, can only be embraced through looking at the technology implications in detail and through understanding the dynamics of the interaction between the various influences.

\section{ACKNOWLEDGMENTS}

This material is based on work supported by Science Foundation Ireland under grant no. 03/CE3/I405.

\section{REFERENCES}

[1] Lehr, B., and Crowcroft, J., "Managing Shared Access to a Spectrum Commons", in Proceedings of IEEE DySPAN 2005, Balitmore, USA, Nov, 2005.

[2] T. Hazlett, The Spectrum-Allocation Debate: An Analysis, in IEEE Internet Computing, September/October 2006, pp 52-58. 
[3] Hatfield, D.N., Weiser, P.J., Property Rights in Spectrum: Taking the Next Step, in proceedings of the IEEE DySPAN 2005, Baltimore, USA, pp 4356.

[4] Coase, R. H., "The Federal Communications Commission," Journal of Law \& Economics, 2 (October 1959), pp. 1-40.

[5] Coase, R.H., "The Interdepartment Radio Advisory Committee," Journal of Law \& Economics, 5 (October 1962), pp. 1747.

[6] De Vany, Arthur, "Implementing a Market-Based Spectrum Policy," Journal of Law \& Economics, 41 (October 1998a), pp. 627-646.

[7] De Vany, Arthur, "Property Rights in the Electromagnetic Spectrum," in Peter Newman, ed., The New Palgrave Dictionary of Economics and the Law, Vol. 3. London: Macmillan, 1998b, pp. 167-171.

[8] De Vany, Arthur S., Ross D. Eckert, Charles J. Meyers, Donald J. O'Hara, and Richard C. Scott, "A Property System for Market Allocation of the Electromagnetic Spectrum: A LegalEconomic-Engineering Study," Stanford Law Review, 21 (June 1969), pp. 1499- 1561

[9] Kwerel, Evan R. and Gregory L. Rosston, ”An Insiders' View of FCC Spectrum Auctions," Journal of Regulatory Economics, 17 (May 2000), pp. 253-289.

[10] Kwerel, Evan R. and John R. Williams, "Free the Spectrum: Market-Based Spectrum Management," in Tom W. Bell and Solveig Singleton, eds., Regulators' Revenge: The Future of Telecommunications Deregulation. Washington, D.C.: Cato Institute, 1998, pp. 101-111.

[11] Kwerel, Evan R. and John R. Williams, "Moving toward a Market for Spectrum," Regulation, 2 (1993), pp. 53-62.
[12] Kwerel, Evan R. and John R. Williams, "Changing Channels: Voluntary Reallocation of UHF Television Spectrum," Office of Plans and Policy Working Paper No. 27. Washington, D.C.: FCC, November 1992.

[13] Kwerel, Evan R. and Alex D. Felker, "Using Auctions to Select FCC Licensees," Office of Plans and Policy Working Paper No. 16. Washington, D.C.: FCC, 1985.

[14] Robert J. Matheson, Flexible Spectrum Use Rights Tutorial, International Symposium of Advanced Radio Technology (2005)

[15] Lawrence J. White, "PROPERTYZING" THE ELECTROMAGNETIC SPECTRUM: WHY IT'S IMPORTANT, AND HOW TO BEGIN, The Progress \& Freedom Foundation Telecommunications Reform Project, October 2000, pp44.

[16] Charles Jackson, Limits to Decentralization: The Example of AM Radio Broadcasting or Was a Common Law Solution to Chaos in the Radio Waves Reasonable in 1927? 1 (2005)

[17] Hamacher, C., "Guard bands for coexistence of UMTS and DVB in a hybrid radio system," IST Mobile Communications Summit 2002, pp. 261-265, 2002.

[18] Forde, T.K, and Doyle, L.E., Externalities, Exclusivities and Easements: Dynamic Spectrum Access and Local Coasean Bargaining, in the Proceedings of the 2nd IEEE Syposium on New Frontiers in Dynamic Spectrum Access Networks, IEEE DySPAN 2007, Dublin, April 18th - 20th, 2007.

[19] Kolodzy, P. Interference Temperature: A Metric for Dynamic Spectrum Utilization, International Journal of Network Management, John Wiley \& Sons, 2006. 